Research article

\title{
Neonatal morphine exposure and maternal deprivation alter nociceptive response and central biomarkers' levels throughout the life of rats
}

\author{
Carla Oliveira $^{\mathrm{a}, \mathrm{b}}$, Roberta Ströher Toledo ${ }^{\mathrm{a}, \mathrm{c}}$, Vanessa Leal Scarabelot ${ }^{\mathrm{a}}$, Rafael Vercelino ${ }^{\mathrm{a}}$, \\ Lisiane Santos da Silva ${ }^{\text {a,d }}$, Gabriela Gregory Regner ${ }^{\mathrm{a}}$, Andressa de Souza ${ }^{\mathrm{a}}$, \\ Natalia Paula Silveira ${ }^{a}$, Wolnei Caumo ${ }^{d}$, Iraci L.S. Torres ${ }^{\mathrm{a}, \mathrm{c}, \mathrm{d}, \mathrm{*}}$

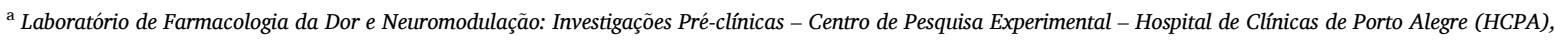 \\ Porto Alegre, RS, Brazil \\ ${ }^{\mathrm{b}}$ Faculdade São Francisco de Assis / UNIFIN, Porto Alegre, RS, Brazil \\ ${ }^{\mathrm{c}}$ Programa de Pós-Graduação em Ciências Biológicas: Farmacologia e Terapêutica-Instituto de Ciências Básicas da Saúde - Universidade Federal do Rio Grande do Sul \\ (UFRGS), Porto Alegre, RS, Brazil \\ d Programa de Pós-Graduação em Ciências Médicas - Universidade Federal do Rio Grande do Sul (UFRGS), Porto Alegre, RS, Brazil
}

\section{A R T I C L E I N F O}

\section{Keywords:}

opioid

maternal-separation

neonate rats

nociception

\begin{abstract}
A B S T R A C T
In the present study, we investigated the effect of repeated neonatal morphine exposure and/or maternal deprivation(MD) on the nociceptive response and central biomarkers' BDNF, IL-1 $\beta$, and IL-4 levels at postnatal days 16(PND16), 30(PND30), and 60(PND60). At birth, the litters were standardized to contain 8 pups/dam $(\mathrm{n}=58)$. From PND1 to PND10, the pups of the deprived groups were separated daily from their mothers for $3 \mathrm{~h}$ and divided into 5 groups: control(C), saline(S), morphine(M), deprived-saline(DS), and deprived-morphine (DM). The pups received subcutaneous injections of saline/morphine $(5 \mu \mathrm{g})$ in the mid-scapular area between PND8 and PND14. Nociceptive responses were assessed by hot plate(HP) and tail-flick(TFL) tests and biomarker levels by ELISA. Thermal hyperalgesia(HP) was found in all assessments for the M, DS, and DM groups, and a decrease in nociceptive threshold(TFL) was found in the DS group at PND16; M and DM groups at PND30; and M, DS, and DM groups at PND60. There were interactions between treatment/deprivation/timepoint in all central biomarkers' levels. The current study indicates that neonatal exposure to morphine and MD, which occurs in the pediatric ICU, can alter the nociceptive and neuroinflammatory responses.
\end{abstract}

\section{Introduction}

Neonatal stress can induce long-term changes in pain sensitivity [1]. Some early sensory experiences can induce alterations in neural development and behavior until adulthood [2]. Maternal deprivation (MD) is an early life stressor which can have lasting effects extending into adulthood, as an alteration in the nociceptive response and the analgesic efficacy, as nociceptive response and analgesic efficacy [3,4].

The nociceptive system in development is especially vulnerable to noxious stimuli [5,6]. Newborn infants hospitalized in a neonatal intensive care unit may experience significant stressors, including repeated painful procedures, as well as MD [7]. Opioid analgesics, such as morphine, are the most effective at reducing pain and stress. They frequently are used in pediatric care despite the concerns about both short- and long-term detrimental effects $[8,9]$. Our group has shown that neonatal morphine exposure promotes long-term changes in nociceptive pathways and alters glutamate signaling and the opioid response in adult life [10-12]. In addition, repeated low-dose morphine administration in neonatal rats induces hyperalgesia in adult life, which could be reversed by a glutamate receptor antagonist [12] or by melatonin administration [13]. MD during childhood induces long-lasting alterations in the regulation of the endogenous opioid system in humans as well as animals $[14,15]$. Our recent studies showed that MD in early life alters neurotrophins and neuroinflammatory cytokines and promotes analgesia in adolescent rats and hyperalgesia in adult rats $[16,17]$.

It is important to highlight that the processing and modulation of nociceptive signals involves the interaction between immunologic mechanisms in the spinal dorsal horn and the transmission of nociceptive information to the brain $[18,19]$. A study by our group showed that early life morphine treatment increases hippocampus BDNF levels, a neurotrophin

\footnotetext{
* Corresponding author at: Hospital de Clínicas de Porto Alegre, Rua Ramiro Barcelos, 2350, 90035-903, Porto Alegre, RS, Brazil.

E-mail address: iltorres@hcpa.edu.br (I.L.S. Torres).
} 
associated with central sensitization in the medium and long-term [20]. Similarly, preclinical studies showed that maternal separation stress alters BDNF levels in limbic areas [21,22]. However, animal studies suggest that morphine may affect immune functions by increasing the secretion of proinflammatory cytokines, such as tumor necrosis factor alpha (TNF- $\alpha$ ), interleukin-6 (IL-6), and interleukin-1beta (IL-1 $\beta$ ) [23,24]. Previous research has demonstrated that neuroinflammatory reactions appear to be an immunological consequence due to early life parental neglect [25,27]. Animals undergoing MD showed an unbalance of the expression of several cytokine genes in the rat brain, such as IL- $1 \beta$ and IL- 4 , suggesting an immunosuppressive response [28]. Accordingly, cytokines contribute to the deregulation of the hypothalamic-pituitary-adrenal axis and promote alterations in neural plasticity, including a decrease in neurotrophic support and an impaired neurogenesis [29,30].

Considering the importance of a deeper understanding of the effects of opioid neonatal analgesia and MD throughout life, the aim of this study was to investigate whether repeated morphine exposure and/or MD alter the nociceptive response over the short, medium, and long term. In addition, we evaluated their repercussions upon the levels of the central biomarkers BDNF, IL-1 $\beta$ and IL-4.

\section{Material and Methods}

\subsection{Animals}

A total of 58 male pups were utilized and were maintained, in the presence of their mothers, in home cages made of polypropylene $(49 \times 34 \times 16 \mathrm{~cm})$ with the floor covered with sawdust. They were maintained under a standard 12-h dark/light cycle (lights on at 7:00a.m. and lights off at 7:00p.m.) in a temperature-controlled environment $\left(22 \pm 2{ }^{\circ} \mathrm{C}\right)$ and had access to water and chow ad libitum. At birth, the male litters were standardized according to Rozisky et al. [10,11] to contain 8 male pups per dam, the same number of breasts of a female rat, to certify that every animal would have equal access to the mother's breast during lactation, thereby providing equivalent nutritional conditions for all pups [31]. On postnatal day 21(PND21), the rats were weaned. All experiments and procedures were approved by the Institutional Committee for Animal Care and Use (GPPG/HCPA \#150614) and conducted in compliance with the Laboratory Guide for the Care and Use of Animals (The National Academies Press, Eighth Edition, 2011). All experiments were conducted under a blind condition and complied with the ethical and methodological standards of the ARRIVE guidelines [32].

\subsection{Experimental Design}

The animals were divided in 5 groups: the total control-group (C), without any intervention; the saline-group (S) which received saline solution; the morphine-group (M) which received morphine; the deprived-saline-group (DS), submitted to MD and received saline solution; and the deprived-morphine-group (DM), submitted to MD and received morphine. The nociceptive responses were assessed in the short (PND16), medium (PND30) and long term (PND60).

\subsection{Maternal Deprivation (MD)}

On PND1, litters were deprived of their mothers for 3 hours/day/10 days $[2,16,17]$. Deprivation consisted of removing the mother from the home cage, and pups were maintained in the original home cage and grouped in the nest in the presence of maternal odor. Cages were transferred to a different room kept at $30-32{ }^{\circ} \mathrm{C}$ to compensate for the loss of the mother's body heat. This protocol alters HPA-axis functionality and stress reactivity later in life and quite consistently causes deficits in cognitive and emotional behavior [33]. Deprivation was carried out between 08:00a.m. and 11:00a.m. Non-deprived rats remained undisturbed in the home cage with their mothers. The first bedding was changed only on PND11 for all groups.

\subsection{Pharmacological Treatment}

Each animal received saline (C, S and DS groups) or morphine administration ( $5 \mu \mathrm{g}$, sc, in the mid-scapular area; $\mathrm{M}$ and DM groups) once daily for 7 days, starting at PND8. At the beginning of the treatment (PND8), the animals weighed an average of $15 \mathrm{~g}$, and the morphine's dose administered was $0.33 \mathrm{mg} / \mathrm{kg}$. However, at the end of treatment (PND14), the animals weighed on average about $22 \mathrm{~g}$, and thus the dose administered was $0.23 \mathrm{mg} / \mathrm{kg}$. This dose was chosen based on previous studies by our research group [12-14,5]. Rats at PND8 have similar neurological development to human newborns[], with a physiologically immature state [34]. This period is characterized by major brain development changes and the plasticity of the nociceptive system [35, 36]. Subcutaneous administration was chosen to avoid the accidental penetration of the viscera, as the pups had very fragile and thin skin. All treatments were administered at 11:00 a.m. Morphine sulfate (Dimorf ${ }^{\circledR}$ $10 \mathrm{mg} / \mathrm{ml}$, Cristália Ltda, São Paulo, Brazil) was provided by Hospital de Clínicas de Porto Alegre, Porto Alegre, Rio Grande do Sul, Brazil.

\subsection{Nociceptive Tests}

\subsubsection{Hot Plate (HP) Test}

Twenty-four hours prior to testing, the animals were habituated to the apparatus for $5 \mathrm{~min}$ to avoid analgesia induced by the novelty [37]. The temperature of the plate was maintained at $55 \pm 0.1{ }^{\circ} \mathrm{C}$, and response was recorded in seconds(s) and a cutoff-time of $20 \mathrm{~s}$ was used to avoid tissue damage.

\subsubsection{Tail-flick latency (TFL) Test}

The animals were exposed to the apparatus to acclimate to the procedure $24 \mathrm{~h}$ prior to the test session [37]. Each animal was placed on the apparatus, and its tail was laid across a nichrome wire coil that was then heated using an electric current. A cutoff-time of $10 \mathrm{~s}$ was used to avoid

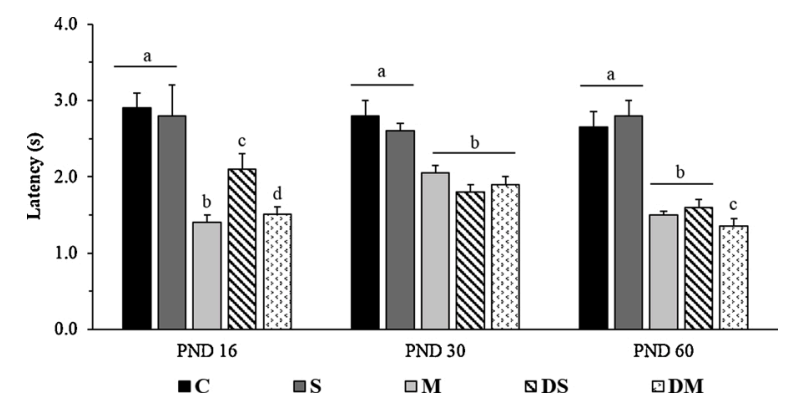

Fig. 1. Hot Plate (HP): Data are presented as mean \pm SEM $(n=58)$. Groups: $C$, control; S, saline; M, morphine; DS, deprived saline; and DM, deprived morphine. Different letters indicate the statistical significance (a-d) between groups at different times.

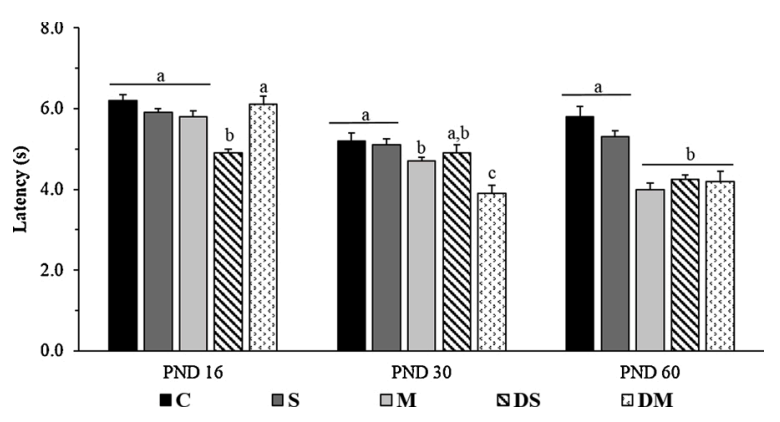

Fig. 2. Tail-Flick (TFL): Data are presented as mean \pm SEM $(n=58)$. Groups: $C$, control; S, saline; M, morphine; DS, deprived saline; and DM, deprived morphine. Different letters indicate the statistical significance (a-c) between groups at different times. 


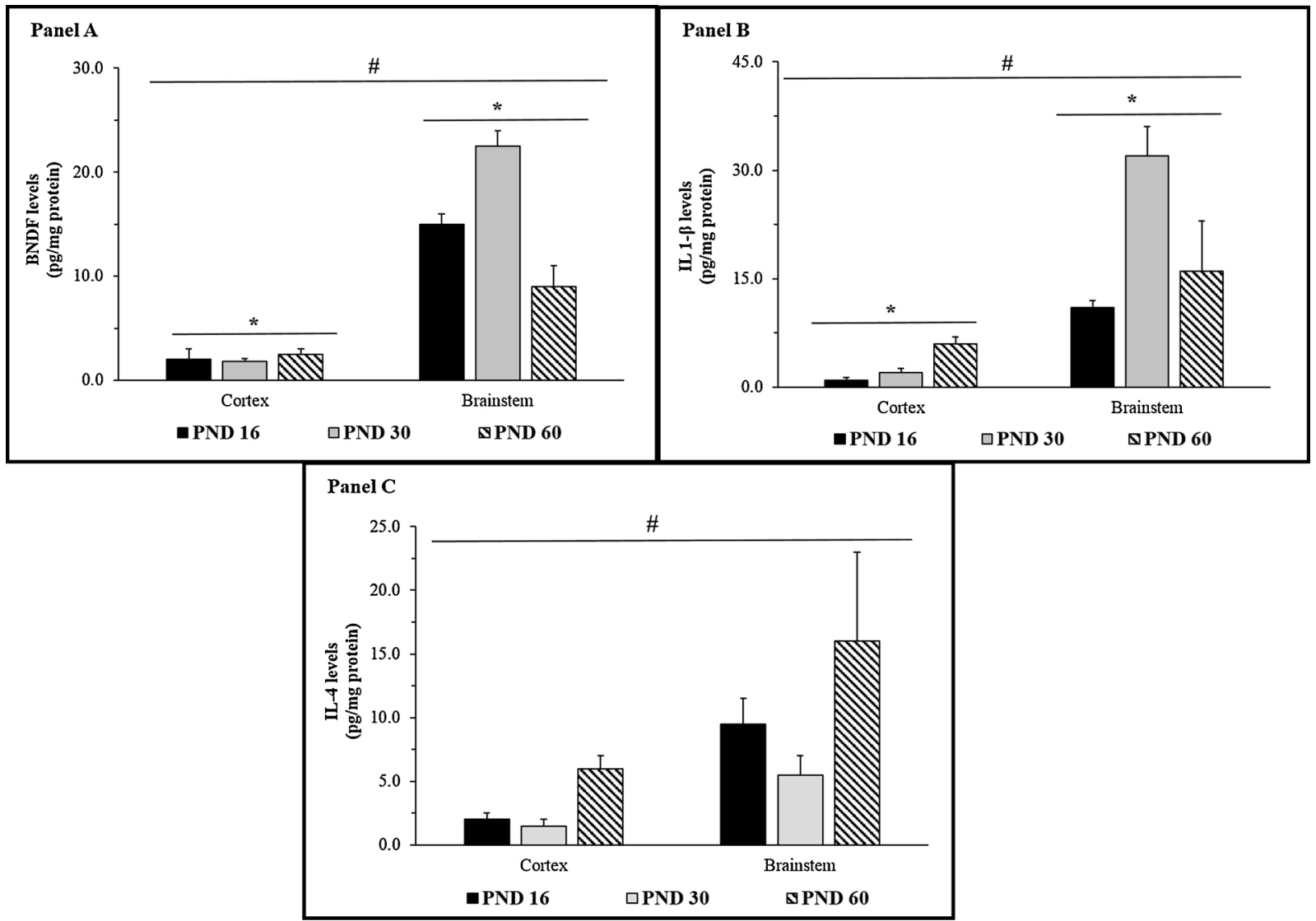

Fig. 3. Baseline Levels of Biomarkers: Data are presented as mean \pm SEM $(n=44)$. Group: C, control. Brainstem and cerebral cortex BDNF, IL-1 $\beta$, and IL-4 levels. Panel A: BDNF levels. "Significant effect of age. ${ }^{\#}$ Interaction between age ${ }^{\times}$structure. Panel B: IL-1 $\beta$ levels. *Significant effect of age. ${ }^{\#}$ Interaction between age ×structure. Panel C: IL-4 levels. ${ }^{*}$ Significant effect of structure.

tissue damage [38]. Three TFL baselines were taken at 3 min intervals.

\subsection{Tissue Collection}

\subsubsection{Biochemical Assays}

The tissue homogenates were prepared with a handheld homogenizer containing Protease Inhibitor Cocktail (Sigma®\#P8340) diluted in phosphate buffered saline (PBS, $\mathrm{pH}$ 7.2) in a proportion of 1:100. Samples were then centrifuged for $5 \mathrm{~min}$ at $10.000 \mathrm{rpm}$, and the supernatant was applied in the BDNF, IL- $1 \beta$ and IL- 4 assays, as determined by sandwich-ELISA, using monoclonal antibodies specific for each measurement (R\&D Systems, Minneapolis, United States). Optical density was measured using an ELISA reader (Hexis - SpectraMax M3) at a wavelength of $450 \mathrm{~nm}$. Each tissue homogenate had its total protein measured by Bradford's method, using bovine serum albumin as standard, and dividing each by its biomarker value. In this way, data were expressed in a $\mathrm{pg} / \mathrm{mg}$ of the protein.

\subsection{Statistical Analysis}

Data were expressed as mean \pm standard error of the mean(SEM). Differences were considered statistically significant if $\mathrm{P}<0.05$. SPSS 20.0 was used for statistical analysis. A Generalized Estimating Equation (GEE) followed by Bonferroni was performed to analyze the results of nociception. If a statistically significant interaction was found, additional pairwise comparisons were made. The significance of the effects was determined by the Wald chi-square statistic. Biochemical data were analyzed by three-way ANOVA followed by the Student Newman-Keuls (SNK) test. To verify the ontogenic effect of biomarkers considering the analyzed structure, control group data were analyzed by two-way
ANOVA followed by the SNK test.

\section{Results}

\subsection{Nociceptive Tests}

\subsubsection{HP Test}

In the HP test, there was an interaction group ${ }^{\mathrm{x}}$ time (Wald $\chi^{2}=32.44$; $8, \mathrm{P}<0.05$ ). Thermal hyperalgesia was indexed by a significant decrease in paw withdrawal latency at PND16, PND30, and PND60 for the animals of the groups M, DS, and DM (Fig. 1).

\subsubsection{TFL Test}

GEE analysis showed an interaction group ${ }^{\times}$time (Wald $\chi^{2}=36.07 ; 8$, $\mathrm{P}<0.05$ ). There was a significant decrease in the nociceptive threshold, as evidenced by the reduction in the latency time in the DS group at PND16, the M and DM groups at PND30, and the M, DS, and DM groups at PND60 (Fig. 2).

\subsection{Biochemical Analysis}

\subsubsection{Biomarkers Baseline Levels}

First, we compared brainstem and cerebral cortex levels of BDNF, IL$1 \beta$ and IL- 4 in the control groups to show that the basal level is different for each brain structure. In relation to the BDNF, we observed an effect of age and interactions between age ${ }^{\times}$structure (two-way ANOVA/SNK, $\mathrm{F}_{(1,38)}=19.20 ; \mathrm{F}_{(1,38)}=26.28$, respectively, $\left.\mathrm{P}<0.05\right)$ (Fig. 3, Panel A). The analysis of IL-1 $\beta$ showed an effect of age and interaction between age $\mathrm{e}^{\mathrm{x}}$ structure (two-way ANOVA/SNK, $\mathrm{F}_{(1,38)}=4.42 ; \mathrm{F}_{(1,38)}=4.45$, respectively $\mathrm{P}<0.05$ ) (Fig. 3 , Panel $\mathrm{B}$ ). In the analysis of $\mathrm{IL}-4$, we 


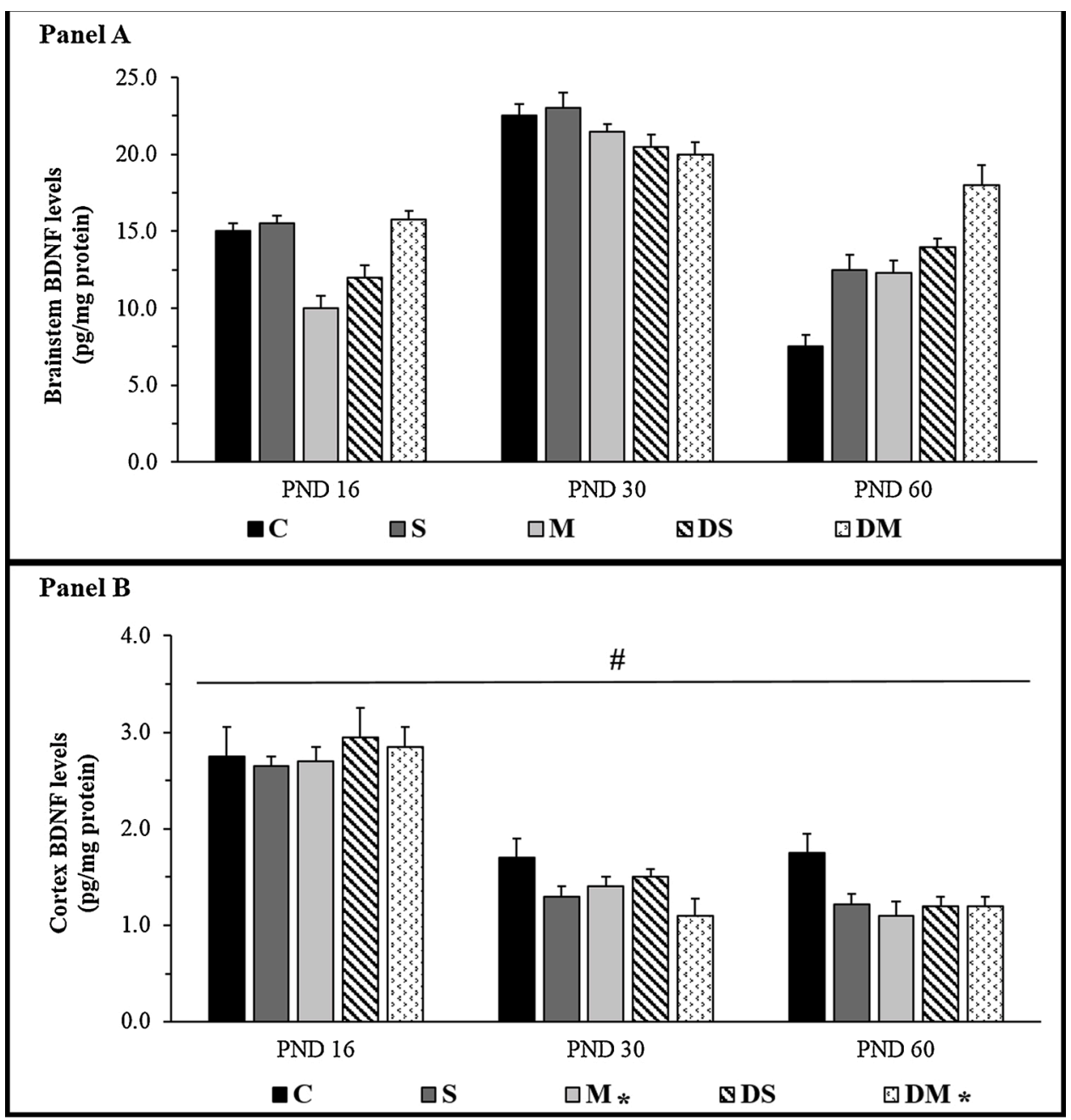

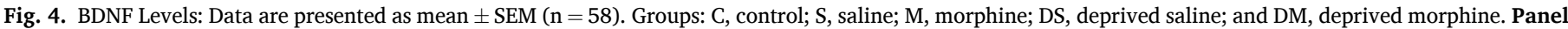
A: Brainstem. *Interaction between treatment ${ }^{\star}$ deprivation, deprivation ${ }^{\star}$ time and treatment ${ }^{\star}$ time. Panel B: Cerebral cortex. *Effect of treatment and \#timepoint.

observed an effect of the structure (two-way ANOVA/SNK, $\mathrm{F}_{(1,38)}=7.39, \mathrm{P}<0.05$ ) (Fig. 3, Panel $\mathrm{C}$ ) on the biomarkers.

\subsubsection{BDNF levels in the brainstem and cerebral cortex}

The brainstem BDNF levels analyses demonstrated interactions between treatment $t^{x}$ deprivation, deprivation ${ }^{x}$ time, and treatment ${ }^{x}$ timepoint (three-way ANOVA/SNK, $\quad \mathrm{F}_{(1,102)}=11.98 ; \quad \mathrm{F}_{(2,102)}=5.24$; $\mathrm{F}_{(4,102)}=4.28$, respectively, $\mathrm{P}<0.05$ ) (Fig. 4, Panel A). There was an effect of treatment in the cerebral cortex levels (three-way ANOVA/SNK, $\left.\mathrm{F}_{(3,103)}=3.43, \quad \mathrm{P}<0.05\right)$ and time (three-way ANOVA/SNK, $\left.\mathrm{F}_{(2,103)}=111.35, \mathrm{P}<0.05\right)$ (Fig. 4, Panel B).

\subsubsection{Brainstem and cerebral cortex $I L-1 \beta$ levels}

In the IL- $1 \beta$ brainstem levels there were interactions between treatment ${ }^{\times}$deprivation, deprivation ${ }^{\times}$time, and treatment ${ }^{x}$ time (three-way ANOVA/SNK, $\mathrm{F}_{(1,103)}=4.37 ; \mathrm{F}_{(2,103)}=7.41 ; \mathrm{F}_{(4,103)}=10.63$, respectively, $\mathrm{P}<0.05$ ) (Fig. 5, Panel A). In the cortex levels, there was an interaction between treatment ${ }^{\times}$deprivation ${ }^{\times}$time (three-way ANOVA/ SNK, $\mathrm{F}_{(2,103)}=3.32, \mathrm{P}<0.05$ ) (Fig. 5, Panel B).

\subsubsection{Brainstem and cerebral cortex IL-4 levels}

We found an interaction between deprivation ${ }^{\times}$time and treatment $\times$ time in brainstem IL-4 levels (three way ANOVA/SNK, $\mathrm{F}_{(2,103)}=4.71$; $\mathrm{F}_{(4,103)}=11.51$, respectively, $\mathrm{P}<0.05$ ) (Fig. 6, Panel A). In the cortex levels, there were interactions between treatment ${ }^{\times}$deprivation ${ }^{\times}$time (three-way ANOVA/SNK, $\left.\mathrm{F}_{(2,103)}=4.72, \mathrm{P}<0.05\right)$ (Fig. 6, Panel B).

\section{Discussion}

The current study shows that both early morphine exposure and MD have significant effects on nociception and central biomarkers throughout the life of the rats. Our data showed that morphine exposure and/or MD induced thermal hyperalgesia in the short (PND16), medium (PND30), and long (PND60) term when evaluated in the HP. Likewise, we observed that MD induced hyperalgesia at PND16, and a decrease in the nociceptive threshold in the TFL induced by morphine exposure and/or MD at PND60.

In the neonatal period, there is great interaction between the mother and the offspring. MD is a stress situation for newborns capable of promoting lasting effects on behavioral responses, including nociception, which can extend into adulthood [16]. There are inconsistent findings in the literature regarding MD and its effects on behaviors, biomarkers, and signaling pathways, possibly due to the different deprivation protocols used in pre-clinical studies (see [39] for review). MD markedly disrupts opioid system development, decreases the analgesic effects of morphine [43,44], and induces an opioid system hypofunction [41,42]. In this way, the hyperalgesia induced by MD found in our study might be related to changes in central opioid activity promoted by this early life stressor $[40,43]$.

From two different methodological nociceptive evaluations, TFL and HP tests, we demonstrated that rats in the neonatal period might be more sensitive to low doses of morphine, since in the first 3 postnatal weeks, there is extensive re-modeling of opioid receptor expression [45]. 


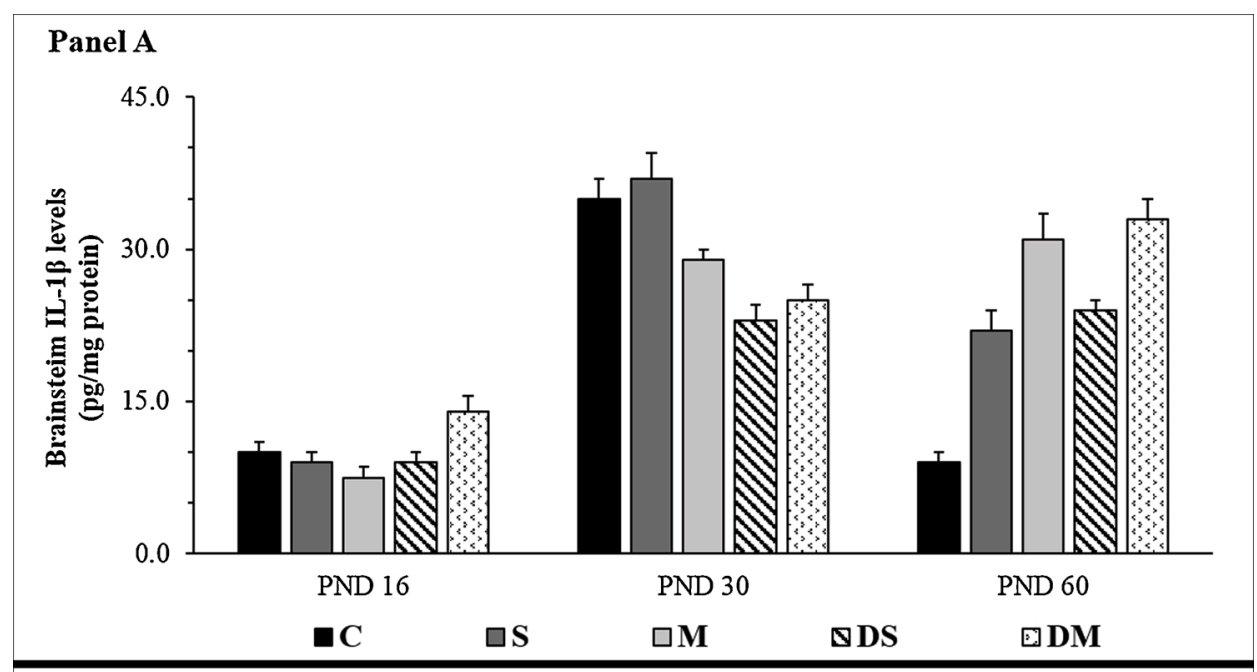

Panel B

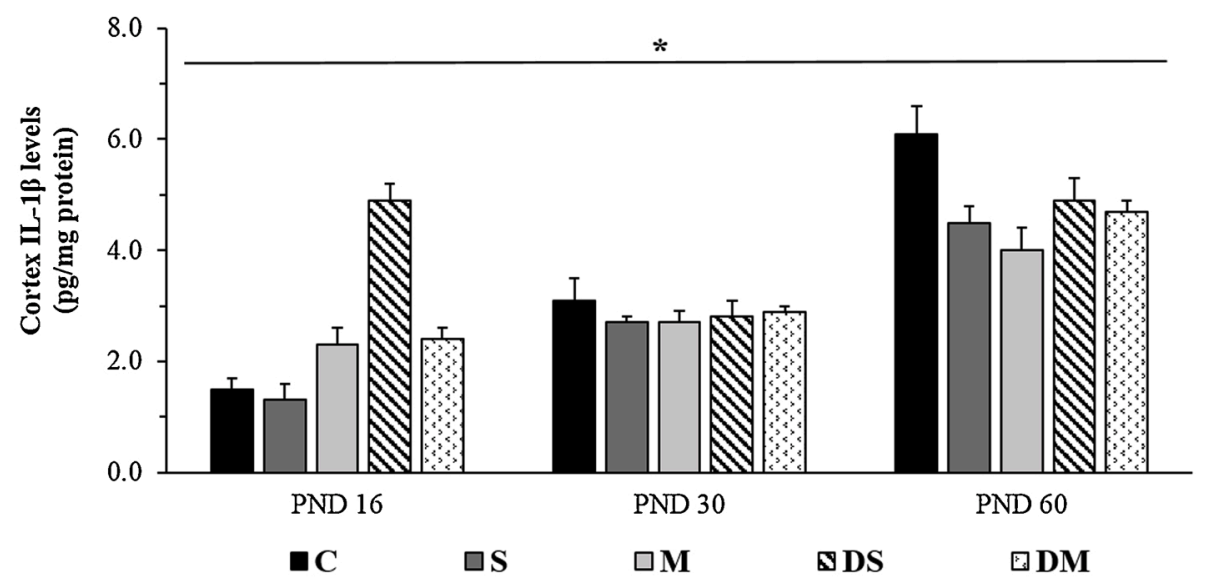

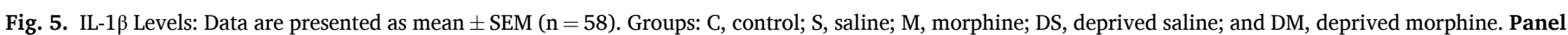

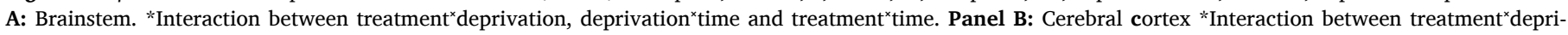
vation ${ }^{\times}$time.

In addition, there is evidence that repeated neonatal opioid exposure leads to spinal cord neuronal hyperactivity, inducing neuroplasticity [46,47] and changes in the supraspinal pain modulatory circuits [48]. Opioids can induce hyperalgesia under many circumstances, which might contribute to the drawbacks of acute and chronic administration of these drugs [48]. Furthermore, chronic exposure to opioids induces functional alteration in the spinal cord neurons that can manifest as neuronal hyperactivity during opiate withdrawal $[49,50]$.

Chronic opioid exposure during infancy may alter the number and sensibility of opioid receptors, altering the development of the CNS and promoting opioid desensitization throughout life [51,52]. These effects may result from a general reorganization in the structure and function of primary afferent synapses, neurotransmitter/receptor expression and function, and excitatory and inhibitory modulation from higher brain centers [34]. Our research group has already demonstrated that morphine exposure during early life can alter the developing nervous system, promoting long-lasting neurochemical and behavioral alterations in rodents $[10,12,53,54]$. The outcomes of the present study, associated with previous data of our research group, suggest that administration of morphine in the first weeks of life, a period of opioid system development, may play an important role in the nociceptive sensitivity of adult rats inducing a long-lasting alteration on the pharmacological, neurochemical and behavioral responses. In addition, it might lead to drug-induced adaptations in the excitatory pain pathways, such as neuroplastic changes at the receptor level, in number and activity $[11,55]$.

We also observed specific-tissue profiles of neuroimmunomodulators linked to early neonatal pain conditions as an ontogenic effect, suggesting an age and site-specific response. The BDNF, IL- $1 \beta$ and IL-4 brainstem levels were higher than the cortex levels, independent of the age. This result corroborates our previous studies where it was possible to observe that BDNF is less expressed in the cerebral cortex than other brain structures in young and adult rats $[16,56]$. In this way, we can suggest the normal pattern of the distribution of these biomarkers in the CNS involves lower expression in the cerebral cortex than in other brain structures. The higher centers of the CNS have the ability to modulate the nociceptive transmission, and a variety of brain regions are involved in this descending modulation and include the frontal lobe, anterior cingulate cortex, insula, and amygdala [57]. Opioid receptors are found throughout the brain and spinal cord and influence many central functions, such as nociception. These receptors are distributed differently within the central and peripheral nervous systems, presenting functional differences in various structures [61]. In the current study, morphine induced long lasting increases in the BDNF brainstem levels, corroborating our previous studies that showed a long-lasting increase in the central BDNF and NGF levels [20,54]. It is important to highlight that NGF seems to be involved in social stress, since studies have shown that changes in its levels result in the development of adequate strategies to face the stressor itself and, thus, survive [58]. MD alters BDNF levels in limbic areas promoting long-lasting changes upon 


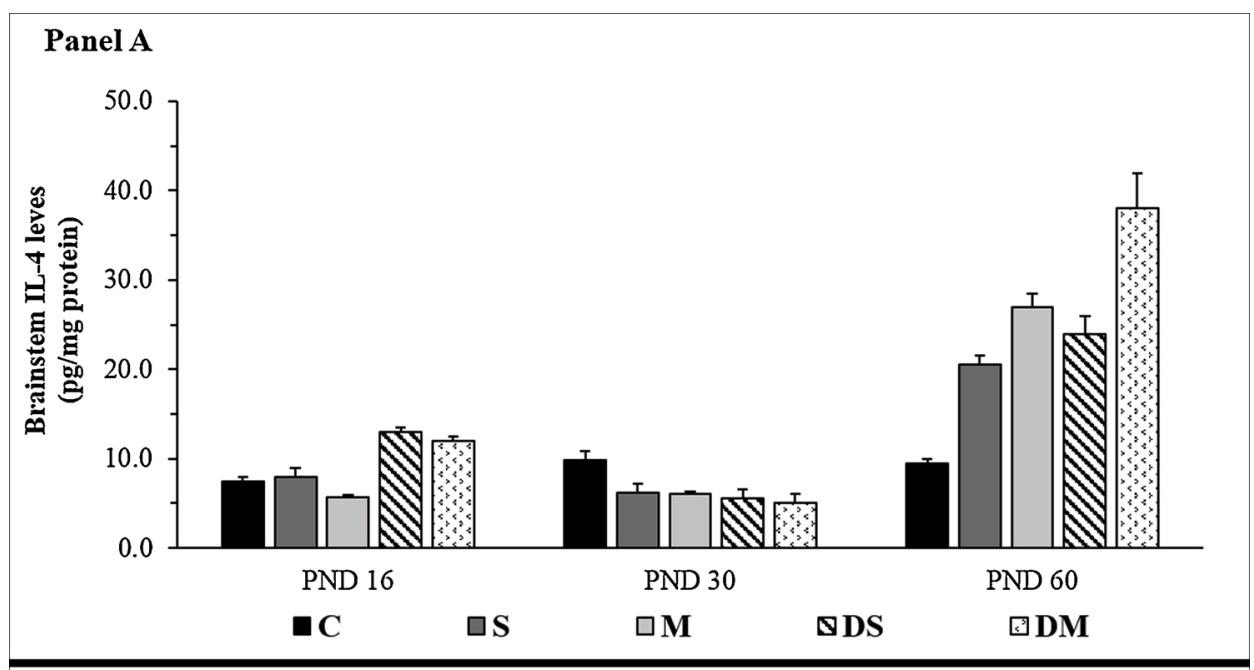

Panel B

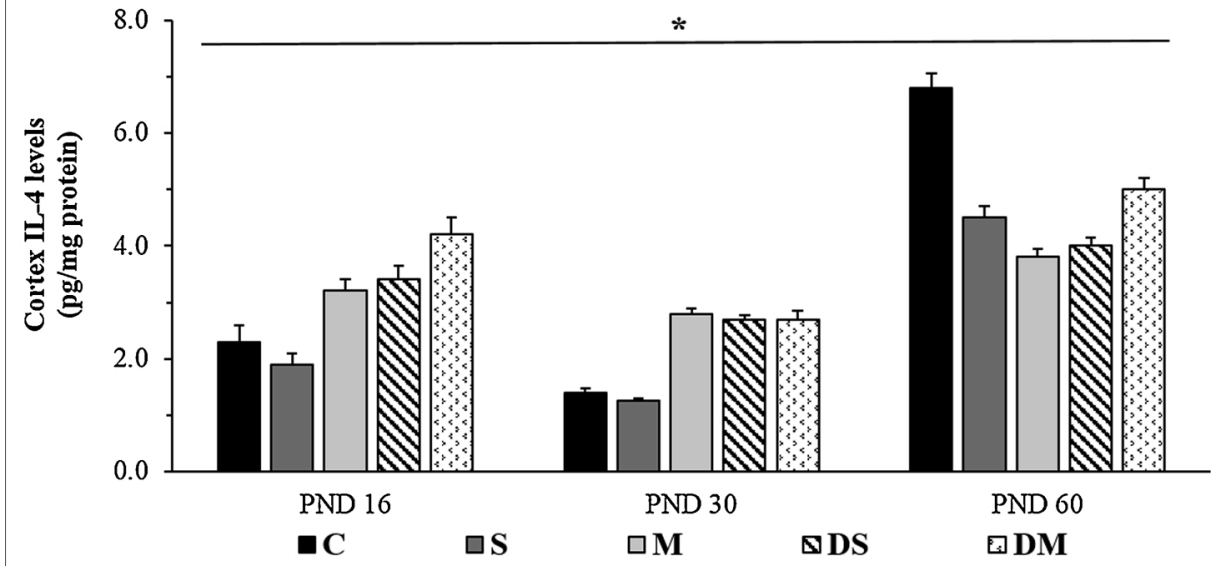

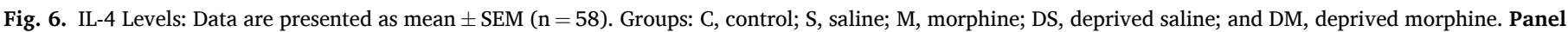
A: Brainstem *Interaction between deprivation ${ }^{\times}$time, and treatment ${ }^{\times}$time. Panel B: Cerebral cortex. "Interaction between treatment ${ }^{\times}$deprivation ${ }^{\times}$time.

emotional behavior and responses to stress [21,22]. This indicates that neurotrophins modulate mechanisms underlying social bonding, and they are sensitive to the handling of the mother-infant relationship and to the rearing environment [21]. Corroborating our results, changes in BDNF expression have been shown to be age, time, and stimulus specific $[17,21]$. In clinical studies, both positive and negative correlations between age and BDNF levels have been reported, which may be due to environmental modulation of the central and peripheral levels $[59,60]$.

We observed that the daily saline injection increased IL- $1 \beta$ brainstem levels, which itself can be considered as an early life stress model. Morphine and MD increased IL- $1 \beta$ and IL- 4 levels in the cortex and brainstem, respectively, and these effects are reverted $b$ associated interventions. Early life challenges alter the phenotype of immune cells, inducing a sensitized state, which can promote an exacerbated reaction later in life, causing greater vulnerability to neurological diseases [61]. Our results corroborate preclinical and clinical studies that show that exposure to negative early life experiences result in alterations to the immune and inflammatory responses, modulating inflammatory processes in adult life $[27,62]$. One limitation of our study was that it was conducted only with male rats, since the nociceptive process and drug responses can be altered by modulations in the hormone state [63].

The current study indicates that neonatal exposure to morphine and to $\mathrm{MD}$, as can occur in a pediatric ICU, can alter the nociceptive and neuroinflammatory responses, which can be related clinical conditions involving chronic pain. This illustrates the need to examine nociceptive processing in human neonate patients that have been exposed to therapeutic morphine and indicates the importance of research evaluating the clinical consequences of long-term opioid administration. In summary, this study emphasizes the importance of conducting research that may counteract opioid and/or MD-induced neuroadaptations to subsequently prevent abnormal pain states and to provide a comprehensive knowledge of their long-lasting effects upon nociceptive and neurochemical pathways.

\section{CRediT authorship contribution statement}

Carla Oliveira: Conceptualization, Investigation, Writing - original draft. Roberta Ströher Toledo: Investigation, Writing - review \& editing. Vanessa Leal Scarabelot: Investigation, Formal analysis. Rafael Vercelino: Validation, Data curation. Lisiane Santos da Silva: Investigation. Gabriela Gregory Regner: Investigation. Andressa de Souza: Resources. Natalia Paula Silveira: Investigation. Wolnei Caumo: Supervision. Iraci L.S. Torres: Methodology, Project administration.

\section{Declaration of Competing Interest}

The authors report no declarations of interest.

\section{Acknowledgements}

This research was supported by the following Brazilian funding agencies: National Council for Scientific and Technological 
Development-CNPq (Dr. Torres ILS; Dr. Caumo W); Brazilian Federal Coordination Agency for the Improvement of Higher Education People CAPES (Toledo RS); Graduate Research Group of Hospital de Clínicas de Porto Alegre - GPPG (Torres ILS-Grant 20150614).

\section{References}

[1] I.P. Butkevich, V.A. Mikhailenko, E.A. Vershinina, et al., Effects of neonatal pain, stress and their interrelation on pain sensitivity in later life in male rats, Chin J Physiol. 59 (4) (2016) 225-231.

[2] F. Benetti, C.K. da Silveira, J. Rosa, et al., Histamine acting on the basolateral amygdala reverts the impairment of aversive memory of rats submitted to neonatal maternal deprivation, Behav Brain Res. 278 (2015) 83-89.

[3] M. Iijima, S. Chaki, Separation-induced ultrasonic vocalization in rat pups: further pharmacological characterization, Pharmacol Biochem Behav. 82 (2005) 652-657.

[4] A.L. Dickinson, M.C. Leach, P.A. Flecknell, Influence of early neonatal experience on nociceptive responses and analgesic effects in rats, Lab Anim. 43 (1) (2009) 11-16.

[5] F. Schwaller, M. Fitzgerald, The consequences of pain in early life: injury-induced plasticity in developing pain pathways, Eur J Neurosci. 39 (2014) 344-355.

[6] S.M. Walker, S. Beggs, M.L. Baccei, Persistent changes in peripheral and spinal nociceptive processing after early tissue injury, Exp Neurol. 275 (2016) 253-260.

[7] S.L. Hays, R.J. McPherson, S.E. Juul, et al., Long-term effects of neonatal stress on adult conditioned place preference (CPP) and hippocampal neurogenesis, Behav Brain Res. 227 (1) (2012) 7-11.

[8] R. Carbajal, R. Lenclen, M. Jugie, et al., Morphine does not provide adequate analgesia for acute procedural pain among preterm neonates, Pediatrics. 115 (2005) 1494-1500.

[9] S. Beggs, Long-term consequences of neonatal injury, Can J Psychiatry. 60 (4) (2015) 176-180.

[10] J.R. Rozisky, G. Dantas, L.S. Adachi, et al., Long-term effect of morphine administration in young rats on the analgesic opioid response in adult life, Int $\mathrm{J}$ Dev Neurosci. 26 (2008) 561-565.

[11] J.R. Rozisky, L.F. Medeiros, L.S. Adachi, et al., Morphine exposure in early life increases nociceptive behavior in a rat formalin tonic pain model in adult life, Brain Res. 1367 (2011) 122-129.

[12] J.R. Rozisky, D. Vendite, F.U. Fontella, et al., Morphine treatment in early life alters glutamate uptake in the spinal synaptosomes of adult rats, Neurosci Lett. 529 (1) (2012) 51-54.

[13] J.R. Rozisky, V.L. Scarabelot, C. de Oliveira, et al., Melatonin as a Potential Counter-Effect of Hyperalgesia Induced by Neonatal Morphine Exposure, Neurosci Lett. 633 (2016) 77-81.

[14] A. Troisi, G. Frazzetto, V. Carola, et al., Variation in themu-opioid receptor gene (OPRM1) moderates the influence of early maternal care on fearful attachment, SocCogn Affect Neurosci. 7 (5) (2012) 542-547.

[15] M. Preter, D.F. Klein, Lifelong opioidergic vulnerability through early life separation: a recent extension of the false suffocation alarm theory of panic disorder, Neurosci Bio behav Rev. 3 (2014) 345-351.

[16] R. Ströher, C. de Oliveira, B.C. Lopes, et al., Maternal Deprivation Alters Nociceptive Response in a Gender-Dependent Manner in Rats, Int J Dev Neurosci. 76 (2019) 25-33.

[17] R. Ströher, C. de Oliveira, D.J. Stein, et al., Maternal Deprivation and Sex Alter Central Levels of Neurotrophins and Inflammatory Cytokines in Rats Exposed to Palatable Food in Adolescence, Neuroscience. 428 (2020) 122-131.

[18] U.K. Hanisch, H. Kettenmann, Microglia: active sensor and versatile effector cells in the normal and pathologic brain, Nat Neurosci. 10 (2007) 1387-1394.

[19] H. Kettenmann, U.K. Hanisch, M. Noda, et al., Physiology of microglia, Physiol Rev. 91 (2011) 461-553.

[20] J.R. Rozisky, G. Laste, I.C. de Macedo, et al., Neonatal Morphine Administration Leads to Changes in Hippocampal BDNF Levels and Antioxidant Enzyme Activity in the Adult Life of Rats, Neurochem Res. 38 (3) (2013) 494-503.

[21] M. Roceri, F. Cirulli, C. Pessina, et al., Postnatal repeated maternal deprivation produces age-dependent changes of brain-derived neurotrophic factor expression in selected rat brain regions, Biol Psychiatry. 55 (2004) 708-714.

[22] F. Cirulli, F. Capone, L.T. Bonsignore, et al., Early behavioural enrichment in the form of handling renders mouse pups unresponsive to anxiolytic drugs and increases NGF levels in the hippocampus, Behav Brain Res. 178 (2007) 208-215.

[23] M.R. Hutchinson, Y. Shavit, P.M. Grace, et al., Exploring the neuroimmunopharmacology of opioids: an integrative review of mechanisms of central immune signaling and their implications for opioid analgesia, Pharmacol Rev. 63 (2011) 772-810.

[24] I.K. Madera-Salcedo, S.L. Cruz, C. Gonzalez-Espinosa, Morphine decreases early peritoneal innate immunity responses in Swiss-Webster and C57BL6/J mice through the inhibition of mast cell TNF-alpha release, J Neuroimmunol. 232 (2011) 101-107.

[25] A. Danese, C.M. Pariante, A. Caspi, et al., Childhood maltreatment predicts adult inflammation in a life-course study, Proc Natl Acad Sci. 104 (2007) 1319-1324.

[27] E. Painsipp, M.J. Köfer, F. Sinner, et al., Prolonged depression like behaviour caused by immune challenge: influence of mouse strain and social environment, PLoS One. 6 (2011) 1-11.

[28] J.J. Dimatelis, N.S. Pillay, A.K. Mutyaba, et al., Early maternal separation leads to down-regulation of cytokine gene expression, Metab Brain Dis. 27 (3) (2012).

[29] D.M. Christmas, J. Potokar, S.J. Davies, A biological pathway linking inflammation and depression: activation of indoleamine 2,3-dioxygenase, Neuropsychiatr. Dis. Treat. 7 (2011) 431-439.
[30] I. Gracia-Rubio, M. Moscoso-Castro, O.J. Pozo, et al., Maternal separation induces neuroinflammation and long-lasting emotional alterations in mice, Prog Neuropsychopharmacol Biol Psychiatry. 4 (65) (2016) 104-117.

[31] T. Tanaka, The relationships between litter size, offspring weight and behavioral development in laboratory mice, Mamm Study Tokio. 29 (2004) 147-153.

[32] C. Kilkenny, W.J. Browne, I.C. Cuthill, et al., Improving bioscience research reporting: the arrive guidelines for reporting animal research, PLoS Biol. 8 (2010) 1000-1412.

[33] S.J. Lupien, B.S. McEwen, M.R. Gunnar, et al., Effects of stress throughout the lifespan on the brain, behaviour and cognition, Nat. Rev. Neurosci. 10 (2009) 434-445.

[34] D. Pattinson, M. Fitzgerald, The neurobiology of infant pain: development of excitatory and inhibitory neurotransmission in the spinal dorsal horn, Reg. Anesth. Pain Med. 29 (1) (2004) 36-44.

[35] T. Rabinowicz, G.M. de Courten-Myers, J.M. Petetot, et al., Human cortex development: estimates of neural numbers indicate major loss late during gestation, Journal of Neuropathology and Experimental Neurology. 55 (1996) $320-328$.

[36] J.J. Kim, M.R. Foy, R.F. Thompson, Behavioral stress modifies hippocampal plasticity through N-methyl-d-aspartate receptor activation, Proceedings of the National Academy of Sciences of the United States of America 93 (1996) $4750-4753$.

[37] C.A. Netto, B. Siegfried, I. Izquierdo, Analgesia induced by exposure to a novel environment in rats: effect of a concurrent and post-training stressful stimulation, Behav Neural Biol. 48 (1987) 304-309.

[38] V.M. Castilho, C.E. Macedo, M.L. Brandão, Role of benzodiazepine and serotonergic mechanisms in conditioned freezing and antinociception using electrical stimulation of the dorsal periaqueductal gray as unconditioned stimulus in rats, Psychopharmacology. 165 (2002) 77-85.

[39] S.G. Tractenberg, M.L. Levandowski, L.A. de Azeredo, et al., An overview of maternal separation effects on behavioural outcomes in mice: Evidence from a four-stage methodological systematic review, Neurosci Biobehav Rev. 68 (2016) 489-503.

[40] S.A. Weaver, J. Diorio, M.J. Meaney, Maternal separation leads to persistent reductions in pain sensitivity in female rats, J Pain. 8 (2007) 962-969.

[41] M. Bernardi, S. Genedani, S. Tagliavini, et al., Effects on long-term sensitivity to pain and morphine of stress induced in the newborn rat by pain or manipulation, Physiol Behav. 37 (5) (1986) 827-831.

[42] C.H. Kwok, I.M. Devonshire, A.J. Bennett, et al., Postnatal maturation of endogenous opioid systems within the periaqueductal grey and spinal dorsal horn of the rat, Pain. 155 (1) (2014) 168-178.

[43] W.F. Sternberg, L. Smith, L. Scorr, Nociception and antinociception during the first week of life in mice: sex differences and test dependence, J Pain. 5 (8) (2004) 420-426.

[44] G. Woolfe, A.D. MacDonald, The evaluation of the analgesic action of pethidine hydrochloride, Journal of Pharmacology and Experimental Therapeutics. 80 (1944) 300-307.

[45] B. Beland, M. Fitzgerald, Mu- and delta opioid receptors are down-regulated in large primary sensory neurons during postnatal development in rats, Pain. 90 (2001) 143-150.

[46] J. Mao, D.J. Mayer, Spinal cord neuroplasticity following repeated opioidexposure and its relation to pathological pain, Ann N Y Acad Sci. 933 (2001) 175-184.

[47] K. Kesavan, Neurodevelopmental implications of neonatal pain and morphineexposure, Pediatr Ann. 44 (2015) 260-264.

[48] M.H. Ossipov, J. Lai, T. King, et al., Underlying mechanisms of pronociceptive consequences of prolonged morphine exposure, Pept Sci. 80 (2005) 319-325.

[49] T.W. Vanderah, N.M. Suenaga, M.H. Ossipov, et al., Tonic descending facilitation from the rostral ventromedial medulla mediates opioid-induced abnormal pain and antinociceptive tolerance, J Neurosci. 21 (2001) 279-286.

[50] L.R. Gardell, T. King, M.H. Ossipov, et al., Opioid receptor-mediated hyperalgesia and antinociceptive tolerance induced by sustained opiate delivery, Neurosci Lett. 396 (2006) 44-49.

[51] R. Hasani, M.R. Bruchas, Molecular Mechanisms of Opioid Receptor-Dependent Signaling and Behavior, Anesthesiology. 11 (6) (2011) 1363-1381.

[52] J.T. Williams, S.L. Ingram, G. Henderson, et al., Regulation of $\mu$-Opioid Receptors: Desensitization, Phosphorylation, Internalization, and Tolerance, Pharmacol Rev. 65 (1) (2013) 223-254.

[53] J.R. Rozisky, G. Laste, L.F. Medeiros, et al., Morphine Treatment in Neonate Rats Increases Exploratory Activities: Reversal by Antagonist D2 Receptor, British Journal of Medicine \& Medical Research. 4 (1) (2014) 351-367.

[54] E.A. Nunes, L.F. Medeiros, J.S. de Freitas, et al., Morphine exposure during early life alters thermal and mechanical thresholds in rats, Int J Dev Neurosci. 60 (2017) $78-85$.

[55] T.L. Yaksh, G.J. Harty, B.M. Onofrio, High Dose of Spinal Morphine Produce a Nonopiate Receptor-Mediated Hyperesthesia: Clinical and Theoretic Implications, Anesthesiology. 64 (5) (1986) 590-597.

[56] S.F.S. Moreira, L.F. Medeiros, A. de Souza, et al., Transcranial direct current stimulation (tDCS) neuromodulatory effects on mechanical hyperalgesia and cortical BDNF levels in ovariectomized rats, Life Sci. 145 (2016) 233-239.

[57] W.Y. Ong, C.S. Stohler, D.R. Herr, Role of the Prefrontal Cortex in Pain Processing, Mol Neurobiol. 56 (2) (2019) 1137-1166.

[58] A. Berry, E. Bindocci, E. Alleva, NGF, Brain and Behavioral Plasticity, Neural Plast. 2012 (2012), 784040.

[59] U.E. Lang, R. Hellweg, J. Gallinat, BDNF serum concentrations in healthy volunteers are associated with depression-related personality traits, Neuropsychopharmacology. 29 (2004) 795-798. 
[60] M. Lommatzsch, D. Zingler, K. Schuhbaeck, et al., The impact of age, weight and gender on BDNF levels in human platelets and plasma, Neurobiol Aging. 26 (2005) $115-123$.

[61] B. Viviani, M. Boraso, M. Valero, et al., Early maternal deprivation immunologically primes hippocampal synapses by redistributing interleukin-1 receptor type I in a sex dependent manner, Brain Behav Immun. 35 (2014) $135-143$.

[62] L.C. Loram, F.R. Taylor, K.A. Strand, et al., Prior exposure to glucocorticoids potentiates lipopolysaccharide induced mechanical allodynia and spinal neuroinflammation, Brain Behav Immun. 25 (2011) 1408-1415.
[63] S. Ribeiro, P. Yang, C. Reyes-Vazquez, et al., Sex differences in tail-flick latency of non-stressed and stressed rats, Int J Neurosci. 115 (10) (2005) 1383-1395.

\section{Further reading}

[26] N. Slopen, T.T. Lewis, T.L. Gruenewald, et al. Early life adversity and inflammation in African Americans and whites in the midlife in the United States survey Psychosom Med. 2010; 72: 694-701. 